\title{
Situs inversus totalis with congenitally corrected transposition of the great arteries: insights from cardiac MRI
}

\author{
Jan M Sohns ${ }^{1,2^{*}}$, Michael Steinmetz ${ }^{2,3}$, Heike Schneider ${ }^{2,3}$, Martin Fasshauer ${ }^{1,2}$, Wieland Staab ${ }^{1,2}$, \\ Johannes Tammo Kowallick ${ }^{1,2}$, Andreas Schuster ${ }^{2,4}$, Christian Ritter ${ }^{1,2}$, Joachim Lotz ${ }^{1,2}$ \\ and Christina Unterberg-Buchwald ${ }^{2,4}$
}

\begin{abstract}
Introduction: Situs inversus totalis with congenitally corrected transposition of the great arteries represents a relatively rare congenital condition.

Case description: The current report describes the case of a 56 year old patient with an atrio-ventricular and ventricular-arterial discordance of the heart chambers without surgical correction, incidentally detected during hepatocellular carcinoma evaluation. The systemic venous blood arrived via the right atrium and a mitral valve in the morphologically left but pulmonary arterial ventricle that gave rise to a pulmonary trunk. The pulmonary venous blood passed the left atrium and the tricuspid valve into a morphologically right but systemic ventricle that gave rise to the aorta.

Discussion and evaluation: The switched anatomy was incidentally detected on echocardiography. The patient was referred to cardiac magnetic resonance imaging (CMR) including flow measurements, volumetry and late enhancement. CMR results showed a mildly impaired function and the switched anatomy. During a follow-up period of 2 years the patient was suffering from only mild heart failure and dyspnea.
\end{abstract}

Conclusions: Heart failure symptoms and arrhythmias can appear with increasing age in patients with congenitally corrected transposition. Early CMR allows accurate diagnosis and timely introduction of adequate therapy thereby avoiding disease progression.

Keywords: Situs inversus; Cardiac MRl; Cardiovascular imaging; Transposition of the great arteries; CMRl; Congential heart disease

\section{Background}

Total situs inversus is a rare diagnosis (Roongruangchai et al. 2012; Iino et al. 2012; Garg et al. 2003; Schmidt et al. 2000; Hoeffel 2001). It can be associated with transposition of the great thoracic arteries (TGA), either of the D- or L-type (i.e. congenitally corrected) TGA (Hoeffel 2001; Warnes 2006; Graham et al. 2000; Patanè et al. 2008; Dyer \& Graham 2003; Shin'oka et al. 2007). The

\footnotetext{
* Correspondence: jan.sohns@med.uni-goettingen.de

'Institute for Diagnostic and Interventional Radiology,

Georg-August-University Göttingen, UMG Universitätsmedizin Göttingen,

Robert-Koch-Str. 40, 37099 Göttingen, Germany

${ }^{2}$ DZHK (German Centre for Cardiovascular Research), partner site Göttingen,

Göttingen, Germany

Full list of author information is available at the end of the article
}

variation of pressure and cardiac workload can damage the myocardium in switched ventricels (Patanè et al. 2008; Dyer \& Graham 2003). Affected patients may develop cardiomyopathy with reduced systolic ejection and arrhythmia if they are not subjected to adequate therapy or early surgery (Hoeffel 2001; Shin'oka et al. 2007). Cardiovascular resonance imaging (CMR) is a powerful non-invasive imaging tool for the characterization of cardiac anatomy in total situs inversus (Schmidt et al. 2000).

\section{Case description}

A 56-year old patient was referred to our center with hepatocellular carcinoma for further diagnostic work-up. Incidentally, an inversion of the liver and abdominal 
structures to the opposite side was seen on computedtomography $(\mathrm{CT})$ and abdominal magnetic-resonance imaging (MRI). During preoperative clinical check-up and postoperative follow-up after partial liver resection (Figure 1), standardized cardiac imaging including Chest Xray and echocardiography were performed. Pre-operative diagnostics demonstrated suspected pulmonary artery hypertension, elevated flow of the atrio-ventricular valve and higher pressure in the supposed right ventricle. Consequently, complete cardiac work-up was performed in the adult congenital heart disease unit.

General clinical examination documented the following findings: The patient suffered from vertigo after getting up fastly and dyspnea during physical exercise. His skin color was rather pale. There was no edema of his legs, and no nycturia was reported. Blood pressure of the right arm was about 130/65 $\mathrm{mmHg}$, and 140/65 $\mathrm{mmHg}$ of the right leg. There was a heart rate around 90 heartbeats (hb) per minute. Nutritional and general conditions were normal. Transcutaneous oxygen saturation was about $100 \%$ and breathing was normal with eupnoea. There were no suspicious breathing sounds of the lungs. The peripheral pulse status was normal.

The electrocardiogram (ECG) at rest showed a normal sinus rhythm with precordial ST- segment depression (V2 to V4), T-wave inversion (V2 to V3) and signs of biventricular hypertrophy.
Echocardiography (Echo) showed a situs inversus totalis with meso- or dextrocardia of the heart. There was a side-by-side position of the great thoracic vessels with suspected atrio-ventricular discordance and ventriculararterial discordance. We detected an L-transposition or congenitally corrected transposition of the great thoracic vessels. The ventricle of the right side (Figure 2) was hypertrophic with more trabecular structures and a typical right ventricular morphology. This ventricle appeared with impaired function. On Echo it was not possible to assess the ejection of this ventricle in detail. There was mild regurgitation of the atrio-ventricular (AV) valve. This AVvalve was located more apically and right-sided.

The ventricle on the left side of the septum appeared as a morphological original left ventricle with less trabecular structures and relatively smooth myocardial wall structures. The AV-valve was located more to the base of the heart and the valve appeared like a morphological mitral valve. The outflow vessel of this ventricle appeared as a pulmonary artery or pulmonary trunk with a low-grade stenosis (peak velocity $1.6 \mathrm{~m} / \mathrm{sec}$ ). There was low-grade AV-valve regurgitation (functional tricuspid valve) and a relatively high right ventricular pressure of $30 \mathrm{mmHg}$. The aortic arch ascended steeply dorsal and was located rather right and medial during descent.

A detailed anatomical view was demonstrated on CMR using ECG-triggered HASTE and True-FISP-
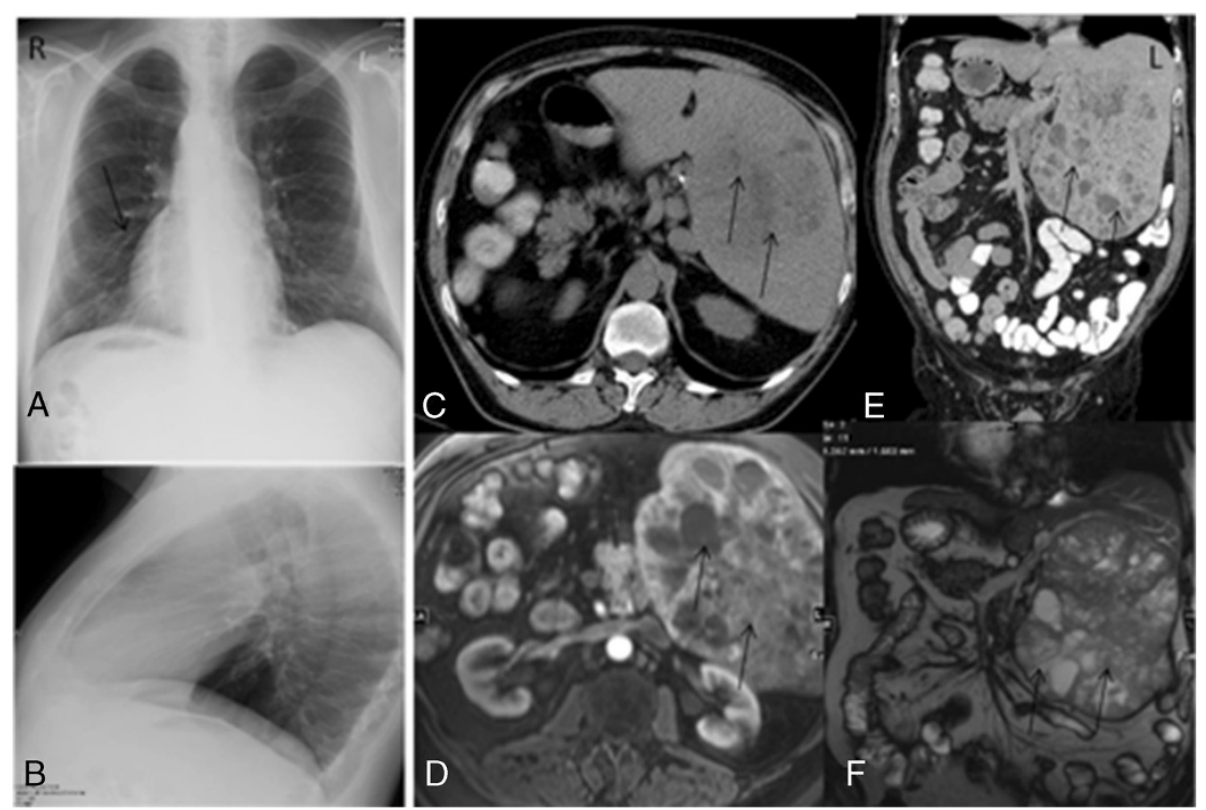

Figure 1 Imaging of situs inversus before CMRI. A: Chest X-ray with dextro-cardia (black arrow), posterior anterior projection, initial image of the first appearance in our hospital in January 2010. B: Lateral view of the chest. C: CT in axial and coronal (E) view after intra-venous contrast medium application showing a situs inversus totalis of the abdomen. The liver is on the left side of this axial view showing a hepatocellular carcinoma infiltrating a liver lobe (black arrows; 64-VCT Light Speed, GE, Healthcare, USA). The same view was seen in transversal (D) and coronal (F) views after application of contrast medium in 1.5 T MRI (Magnetom Symphony, Siemens AG, Healthcare sector, Erlangen, Germany). C: Coronal view in CT and MRI with contrast medium. The spleen was right sided (not demonstrated on these slices). 


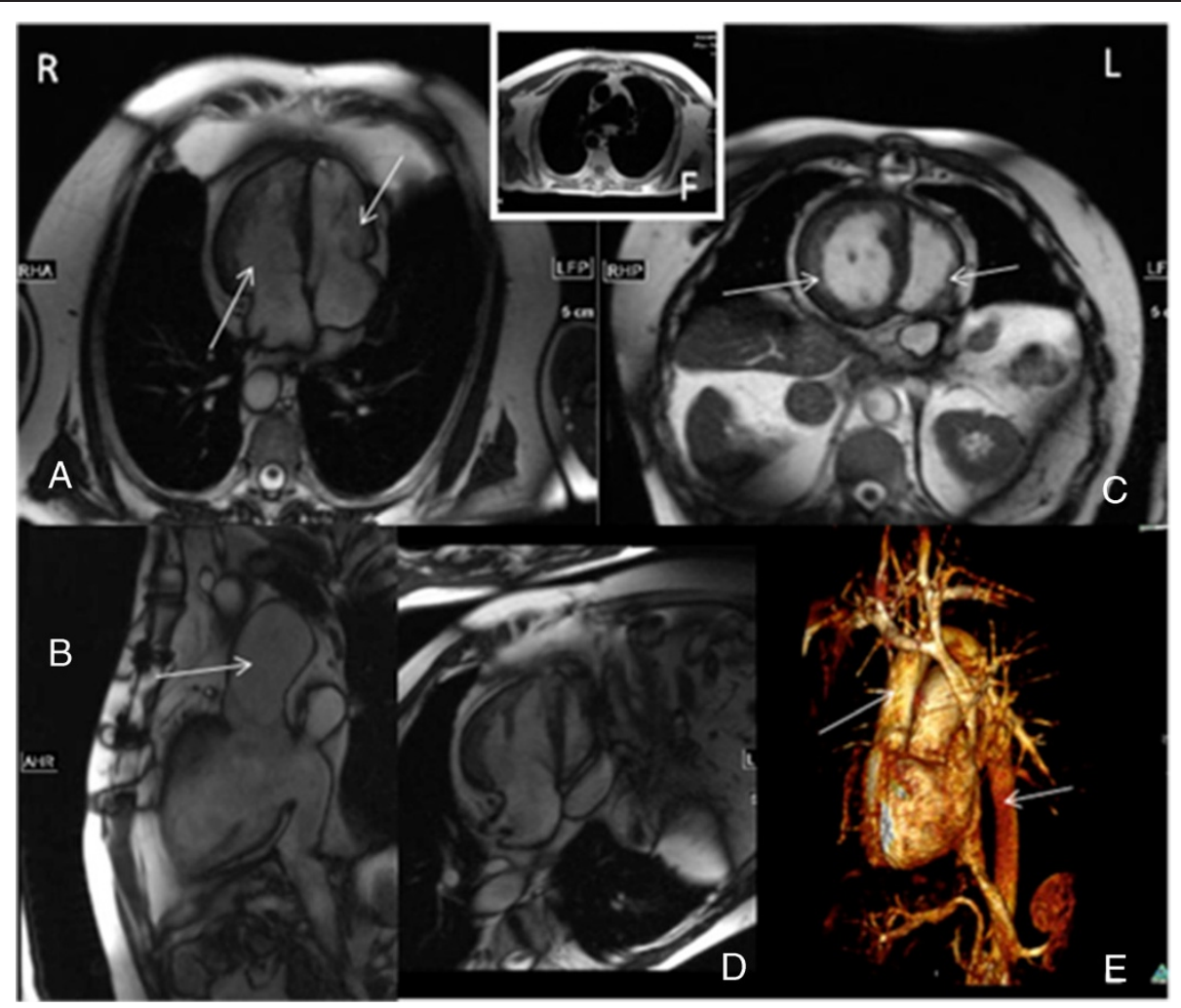

Figure 2 Situs inversus in CMRI. 1.5 T MRI (Magnetom Symphony, Siemens, Healthcare sector, Erlangen, Germany) after application of contrast medium. A: Four-chamber-view with a morphologic right and systemic ventricle (with hypertrophic trabecular structures) as well as a small smoother sub-pulmonary ventricle on the left side (white arrows). B: A dilated pulmonary artery (white arrow) is detected (A). C: Short axis or two-chamber-view (white arrows) demonstrating the cardiac ventricular anatomy of the two ventricles. A D-shaped septum bulging from the systemic right ventricle towards the morphological left ventricle connecting to the pulmonary circulation can be appreciated. D: The outflow of the aortic arch is shown in this image, coming from the functional left ventricle, morphological (original) right ventricle. E: The three-dimensional reconstruction shows the left-sided aorta and right-sided pulmonary trunk (white arrows). F: This image demonstrates the dilated pulmonary trunk and proximal pulmonary arteries in the initial Haste-sequences.

sequences and a segmental approach. The following features were found: The pulmonary veins drained into a morphologically left atrium. The atrio-ventricular valve connecting the left atrium to the ventricle was tricuspid-shaped and located more to the apex, with moderate regurgitation. A hypertrophic ventricle with increased trabeculation, reduced ejection fraction, a moderator band and an infundibulum below the arising great vessel was identified as a morphologically right but functionally systemic ventricle. It gave rise to an anteriorly positioned aorta on the right side, with steeply ascending aortic arch to the dorsal side. The blood from the right upper body side flowed relatively normal via a transversal vein into the left-sided superior vena cava. There was no persisting right superior vena cava. Following the caval veins, the blood drained into a morphologically right atrium, which was connected to a mitral-shaped atrio-ventricular valve. The valve was located more to the base of the heart. The lateral and medial leaflets of the valve were asymmetric with pointed leaflets of the medial valve parts. A smoothly muscled, compact ventricle was connected to this valve, identified as a morphologically left, functionally pulmonary-arterial ventricle. The pulmonary artery was located on the left side related to the aorta and preceded dorsal. The pulmonary arteries were dilated, which can also be seen in patients with pulmonary hypertension or after pulmonary artery embolism. Pulmonary hypertension can be a limiting factor in these patients with increasing heart failure (Roongruangchai et al. 2012; Shin'oka et al. 2007).

The morphologic right, systemic ventricle had an enddiastolic volume of $179 \mathrm{ml}$ (normal: 101-236 ml) and an ejection fraction of $47 \%$ (normal: $55-74 \%$ ).

The morphologic left, sub-pulmonary ventricle had an end-diastolic volume of $83 \mathrm{ml}$ (normal: 110-243 $\mathrm{ml}$ ), an ejection fraction of $53 \%$ (normal: $47-63 \%$ ).

\section{Discussion and evaluation}

The patient had a congenital heart defect with situs inversus totalis and atrio-ventricular and ventriculararterial discordance. 
L-transposition of the great thoracic vessels is very rare (Roongruangchai et al. 2012; Iino et al. 2012; Garg et al. 2003; Schmidt et al. 2000; Hoeffel 2001). This congenital cardiac defect remains often asymptomatic until adulthood (Hoeffel 2001; Warnes 2006; Graham et al. 2000; Patanè et al. 2008; Dyer \& Graham 2003; Shin'oka et al. 2007). When patients are older, heart failure and cardiac arrhythmias can appear. This is caused by progressing failure of the morphological right systemic ventricle that is burdened with high systemic pressures (Schmidt et al. 2000; Shin'oka et al. 2007). A mildly reduced function was already detected in this patient of the right-sided systemic ventricle.

After a pulmonary embolism in September 2010 the patient received Phenprocoumon (INR-adapted 2-2.5). The patient attends frequent outpatient clinics in the unit for adults with congenital heart defects in our hospital with regular CMR scanning. After an interval of two years, in November 2012, after the initial CMR in January 2010, the global function of the heart was stable on CMR and Echo. Whilst the patient had complained about increasing dyspnea at rest, the actual ejection fraction $(11 / 2012)$ was however stable with $45 \%$ (47\% in $1 / 2010)$ of the systemic ventricle (morphologic right) and 62\% (53\% in 1/2010) of the morphologic left (pulmonary arterial ventricle).

It is important to consider the presence of such defects in the adult population. This is underpinned by the fact that the patient had undergone an invasive closure of an atrial-septal defect in 1972 (secundum type). At this time, the situs inversus and the unusual heart anatomy were - surprisingly - not detected and no specific diagnostics followed.

\section{Conclusion}

This case describes the changes in a mid-aged patient with an atrio-ventricular and ventricular-arterial discordance of the heart chambers (L-Transposition of the Great Arteries or congenitally corrected TGA (ccTGA)) without surgical correction. Early CMR allows accurate diagnosis and monitoring of disease progression. The information that can be derived from CMR is useful for timely introduction of adequate therapy allowing optimal treatment with improved quality of life in patients with incidentally detected congenital heart defects.

\section{Patient's consent}

Written informed consent was obtained from the patient for publication of this case report and any accompanying images. A copy of the written informed consent is available for review by the Editor of this journal.

\section{Abbreviation}

AV: Atrio-ventricular; CMRI: Cardiac resonance imaging; CT: Computed tomography; ECG: Electrocardiography; Echo: Echocardiography; HASTE: Half
Fourier Acquisition Single Shot Turbo Spin Echo; MRI: Magnetic resonance imaging; TGA: Transposition of the Great Arteries; True-FISP: True fast imaging with steady state precession.

\section{Competing interests}

The authors declare that they have no competing interests.

\section{Authors' contributions}

JMS wrote the manuscript, did CMRI description and suggestions. MS is a clinician who took care for the general clinical management and revised the manuscript with regard to adult congenital heart disease. HS is a clinician who took care for the general clinical management. MF participated in manuscript creation, gave advices. WS gave advices for correct clinical management and MRI interpretation. JTK did essential comments to the revisions. AS advised on clinical procedures and MRI in congential heart defects. CR did essential improvements of the case description. JL participated in manuscript creation, gave advices. CUB is the senior author who overviewed all steps and gave advices. All authors read and approved the final manuscript.

\section{Author details}

${ }^{1}$ Institute for Diagnostic and Interventional Radiology, Georg-August-University Göttingen, UMG Universitätsmedizin Göttingen, Robert-Koch-Str. 40, 37099 Göttingen, Germany. 'DZHK (German Centre for Cardiovascular Research), partner site Göttingen, Göttingen, Germany. ${ }^{3}$ Department of Pediatric Cardiology and Intensive Care Medicine, Georg-August-University, Göttingen, Germany. ${ }^{4}$ Department of Cardiology and Pneumology, Georg-August-University Göttingen, Göttingen, Germany.

Received: 2 May 2014 Accepted: 30 September 2014

Published: 15 October 2014

\section{References}

Dyer K, Graham TP (2003) Congenitally corrected transposition of the great arteries: current treatment options. Curr Treat Options Cardiovasc Med 5(5):399-407

Garg N, Agarwal BL, Modi N, Radhakrishnan S, Sinha N (2003) Dextrocardia: an analysis of cardiac structures in 125 patients. Int J Cardiol 88(2-3):143-156

Graham TP Jr, Bernard YD, Mellen BG, Celermajer D, Baumgartner H, Cetta F, Connolly HM, Davidson WR, Dellborg M, Foster E, Gersony WM, Gessner IH, Hurwitz RA, Kaemmerer H, Kugler JD, Murphy DJ, Noonan JA, Morris C, Perloff JK, Sanders SP, Sutherland JL (2000) Long-term outcome in congenitally corrected transposition of the great arteries: a multi-institutional study. J Am Coll Cardiol 36(1):255-261

Hoeffel JC (2001) Congenitally corrected transposition of the great arteries (L-TGA) with situs inversus totalis in adulthood: findings with magnetic resonance imaging. Magn Reson Imaging 19(5):762

lino K, Watanabe G, Ishikawa N, Tomita S (2012) Total endoscopic robotic atrial septal defect repair in a patient with dextrocardia and situs inversus totalis. Interact Cardiovasc Thorac Surg 14(4):476-477

Patanè S, Marte F, Di Bella G (2008) Transposition of the great arteries. Int J Cardiol 130(3):98-99

Roongruangchai J, Narongsak W, Plakornkul V, Viravud Y, Sripaoraya K, Roongruangchai K (2012) Situs inversus totalis and ultrastructure of respiratory cilia: report of a cadaveric case. J Med Assoc Thai 95(1):132-138

Schmidt M, Theissen P, Deutsch HJ, Dederichs B, Franzen D, Erdmann E, Schicha $H$ (2000) Congenitally corrected transposition of the great arteries (L-TGA) with situs inversus totalis in adulthood: findings with magnetic resonance imaging. Magn Reson Imaging 18(4):417-422

Shin'oka T, Kurosawa H, Imai Y, Aoki M, Ishiyama M, Sakamoto T, Miyamoto S, Hobo K, Ichihara Y (2007) Outcomes of definitive surgical repair for congenitally corrected transposition of the great arteries or double outlet right ventricle with discordant atrioventricular connections: risk analyses in 189 patients. J Thorac Cardiovasc Surg 133(5):1318-1328

Warnes CA (2006) Transposition of the great arteries. Circulation 114(24):2699-2709, 12

\section{doi:10.1186/2193-1801-3-601}

Cite this article as: Sohns et al:: Situs inversus totalis with congenitally corrected transposition of the great arteries: insights from cardiac MRI. SpringerPlus 2014 3:601. 